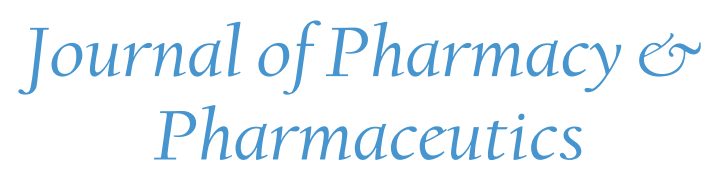

\title{
Linezolid for Methicillin-Resistant Staphylococcus aureus Pneumonia: The Impact of Obesity on Clinical Outcomes
}

\author{
Young R. Lee ${ }^{*}$, Kathy Tang ${ }^{2}$, Rene M. Mani ${ }^{3}$ \\ ${ }^{1}$ Texas Tech University Health Sciences Center, School of Pharmacy, Pharmacy Practice Department, USA \\ ${ }^{2}$ Clinical Pharmacist, Hendrick Medical Center, USA \\ ${ }^{3}$ Clinical Pharmacy Specialist, Hematology-Oncology, Dallas VA Medical Center, USA
}

"Corresponding author: Young R. Lee, Pharm.D., BCPS, BCCCP, Texas Tech University Health Sciences Center, School of Pharmacy, Pharmacy Practice Department, 1718 Pine Street, Abilene, TX 79601, USA, Tel: +1-325-696-0449;

Email: young.lee@ttuhsc.edu

\begin{abstract}
Objectives: Linezolid is an antibiotic used for the treatment of methicillin-resistant Staphylococcus aureus (MRSA) pneumonia. Previous studies provided conflicting results regarding fixed-dose linezolid for obese patients with MRSA pneumonia. Some reported decreased concentrations in obese patients, while others reported similar concentrations and clinical outcomes. This study compared clinical efficacy and safety outcomes between obese and non-obese patients treated with linezolid for MRSA pneumonia.

Methods: This was a retrospective chart review of adults who received at least 3 days of linezolid for MRSA pneumonia from October 1, 2009 to December 31, 2014. Primary outcome was clinical cure rate at 21 days. Secondary outcomes included inpatient mortality, thrombocytopenia, and anemia. Categorical and continuous data were evaluated using the Fisher's exact test and the student's t test, respectively. A p-value $<0.05$ was considered statistically significant.

Results: Forty-five patients in the non-obese group [body mass index (BMI) $<30$ $\mathrm{kg} / \mathrm{m}^{2}$ ], and 25 patients in the obese group [BMI $\geq 30 \mathrm{~kg} / \mathrm{m}^{2}$ ] were identified. Clinical cure rate was $60 \%$ in the non-obese group and $48 \%$ in the obese group. Results of the secondary outcomes were reported as follows: anemia (64\% in the non- obese group, $31 \%$ in the obese group), thrombocytopenia ( $40 \%$ in the non-obese group, $20 \%$ in the obese group), and in-hospital mortality ( $20 \%$ in the non-obese group, $15.6 \%$ in the obese group).

Conclusions: Similar incidence rates of clinical cure, mortality, thrombocytopenia, and anemia were found between obese and non-obese patients who received standard dose linezolid for MRSA pneumonia.
\end{abstract}

Received date: May 02, 2016

Accepted date: September 20, 2016

Published date: September 26, 2016

Citation: Lee, Y.R., et al. Linezolid for Methicillin-Resistant Staphylococcus aureus Pneumonia: The Impact of Obesity on Clinical Outcomes. (2016) J Pharm Pharmaceutics 3(2): 90- 95 .

DOI: $10.15436 / 2377-1313.16 .022$

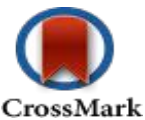

Keywords: Methicillin-resistant Staphylococcus aureus; Pneumonia; Obesity; Oxazolidinones

\section{Introduction}

Overall prevalence of invasive Methicillin-resistant Staphylococcus aureus (MRSA) infections has stabilized or decreased slightly over the past several years since the first isolate was described in the early $1960 \mathrm{~s}^{[1-3]}$. While initially only related to healthcare exposure, such infections started to emerge in community-dwelling individuals in the mid-1990s. In recent years, MRSA epidemiology has become increasingly complex due to co-mingling of community-acquired and healthcare-associated strains in both community and healthcare settings ${ }^{[1]}$. Specifically, the frequency of MRSA pneumonia ranges from $8.9 \%$ (community-acquired pneumonia)

Copyrights: (C) 2016 Lee, Y.R. This is an Open access article distributed under the terms of Creative Commons Attribution 4.0 International License. 
to $26.5 \%$ (healthcare-associated pneumonia) of culture-positive cases of the various pneumonia syndromes ${ }^{[4]}$. In 2015, Centers for Disease Control and Prevention reported that pneumonia led to 16.9 deaths per 100,000 populations and 3.3 in-hospital deaths per 100 discharges ${ }^{[5]}$. The Infectious Diseases Society of America recommends either vancomycin or linezolid as initial therapy, with clindamycin as an alternative agent, for cases of hospital-acquired or community-acquired pneumonia that have suspicious signs and symptoms of MRSA infection or if there is a high incidence of MRSA locally ${ }^{[1,6,7]}$.

Linezolid is a member of the oxazolidinone class and is approved by the Food and Drug Administration for the treatment of both hospital-acquired and community-acquired pneumonia caused by $\mathrm{MRSA}^{[8]}$. The recommended treatment dose of linezolid is fixed at $600 \mathrm{mg}$ orally or intravenously every 12 hours. It is unknown if this dose is sufficient for treating MRSA pneumonia in obese patient populations. This is a pressing clinical concern, as more than $30 \%$ of adult men and women in the United States were classified as obese in the year 2009-2010 (obesity defined as body mass index $\left.[\mathrm{BMI}] \geq 30 \mathrm{~kg} / \mathrm{m}^{2}\right)^{[9]}$. Obesity is a known risk factor for several comorbid diseases and has been associated with impaired immune function and increased infection-related morbidity and mortality ${ }^{[10]}$. Physiologic changes in obesity, such as delayed gastric emptying, altered hepatic metabolism, and increased renal clearance result in pharmacokinetic changes for antimicrobial agents; such changes may result in inadequate antimicrobial exposure and delayed or decreased clinical efficacy ${ }^{[10]}$.

There is a paucity of literature describing appropriate dosing of antibiotics in obesity; with data specific to linezolid, there are many varied results and conflicting conclusions. In a pharmacokinetic study by Bhalodi et al., the authors suggested dose adjustment was not needed in obese patients ${ }^{[11]}$. There were 20 obese patients in the study and no significant differences were found in linezolid concentrations between the moderately and morbidly obese groups. The study concluded standard dosing in patients weighing up to $150 \mathrm{~kg}$ should provide equivalent drug exposure. In contrast, two independent pharmacokinetics studies by Hamilton et al. and Stein et al. found significant differences in linezolid concentration in obese patients compared to nonobese patients ${ }^{[12,13]}$. Hamilton et al. concluded that the average linezolid concentration was more than $50 \%$ lower in the pre-surgery period compared to the post-surgery period in patients with gastric bypass surgery ${ }^{[12]}$. Their findings suggested lower effectiveness with standard doses of linezolid in obese patients. Stein et al. reported diminished blood concentrations of linezolid in seven outpatients (all patients had a total body weight greater than $50 \%$ of ideal body weight) for the treatment of cellulitis; however, they also reported prolonged serum inhibitory activity against all but the least susceptible strain of MRSA ${ }^{[13]}$.

Additionally, several case reports have described reduced linezolid blood concentrations in obese patients ${ }^{[14-16]}$. In the case report by Tsuji et al., the authors suggested linezolid may be highly metabolized in obese patients, which results in lower concentrations of linezolid ${ }^{[15]}$. Mersfelder et al. determined a dosing correction factor (0.27) for linezolid in a morbidly obese patient (BMI $\left.86 \mathrm{~kg} / \mathrm{m}^{2}\right)^{[16]}$. Another case report described decreased effectiveness of linezolid in a morbidly obese male (BMI $82 \mathrm{~kg} / \mathrm{m}^{2}$ ) with community-acquired MRSA pneumonia; this patient had low linezolid concentrations and required addi- tional treatment (vancomycin) due to persistent MRSA from endotracheal aspirate culture and fevers after 48 hours of linezolid treatment ${ }^{[17]}$. On the other hand, a recently published secondary analysis of data from two prospective phase IV trials of 224 patients with MRSA hospital-acquired pneumonia treated with linezolid found that clinical success rates and side effects were similar across all weight categories (weight range from $40 \mathrm{~kg}$ to $215 \mathrm{~kg}$ divided into 4 groups $)^{[18]}$.

With regard to potential side effects, a phase I clinical trial reported that a decrease in red and white blood cell counts may occur shortly after linezolid administration and persist for up to 24 hours; Stein et al. reported that the degree of this side effect may differ between patients of different weights, potentially requiring linezolid dose adjustment ${ }^{[19]}$.

Even though many case reports and studies indicate decreased linezolid concentrations, due to the conflicting clinical outcomes, it is inconclusive whether the efficacy of linezolid among obese patients is similar to non-obese patients. Hence, the purpose of this study was to compare clinical efficacy and safety outcomes between obese and non-obese patients treated with linezolid for MRSA pneumonia.

\section{Patients and Methods}

This single-center retrospective study was conducted at a 522-bed non-profit community hospital in West Texas. Patients were enrolled in this study if they had a documented indication of bacterial pneumonia, documented culture results positive for MRSA, treatment with linezolid $600 \mathrm{mg}$ orally or intravenously (IV) twice daily for at least 6 doses (3 days), and were admitted to the study hospital between October 1, 2009 and December 31,2014 . Patients were excluded if they were less than 18 years of age, lacked any documented indication for linezolid therapy or documented culture results, received less than 3 days or full therapeutic dose of linezolid, or were pregnant or incarcerated at the time of hospital admission. Patients were screened for eligibility and subsequently enrolled. They were placed into either the obese group (BMI $\geq 30 \mathrm{~kg} / \mathrm{m}^{2}$ ) or the non-obese group (BMI $<30 \mathrm{~kg} / \mathrm{m}^{2}$ ). Prior to study commencement, approval was granted by the institutional review boards of Texas Tech University Health Sciences Center and Hendrick Medical Center, the latter being the study site.

Patients were screened for study eligibility by using pharmacy billing code for linezolid (IV or oral). IV and oral linezolid were not differentiated in this study since linezolid has excellent bioavailability $(\sim 100 \%)$. Data were collected by means of electronic or paper chart review. A data collection form was used to include the following: patient demographics (age, gender, weight, height, BMI), in-hospital mortality, pertinent microbiologic data (sputum culture results, vancomycin MIC), pertinent laboratory data (serum creatinine, complete metabolic panel, WBC, hemoglobin, platelets), presence of infiltrate on chest X-ray, number of linezolid doses, utilization of antibiotics other than linezolid (concomitantly or within the past 7 days), presence of concomitant use of antipyretic or corticosteroid therapy, and components of the Simplified Acute Physiology Score II (SAPS II) for those patients who were admitted to the intensive care unit. Creatinine clearance was calculated for each patient using the Cockcroft-Gault formula.

The primary endpoint in this study was clinical cure 
rate at day 21. Secondary endpoints included incidence of anemia, incidence of thrombocytopenia, and in-hospital mortality. Clinical cure was defined as meeting the following criteria: lack of progression of infection, resolution of pneumonia on chest $\mathrm{x}$-ray, no further antibiotic therapy needed, and afebrile status ${ }^{[1]}$. Patients who were discharged before day 21 were classified as clinically cured if they did not require further linezolid treatment after discharge and did not return to the hospital with a subsequent episode of pneumonia within 30 days.

Clinical failure was defined as worsening signs and symptoms of pneumonia after at least 2 days of treatment, imaging indicative of worsening of disease due to pneumonia, development of new pulmonary or extra pulmonary clinical findings that are consistent with active infection, and change in antibiotics or development of a new infection ${ }^{[1]}$. Anemia was defined as a hemoglobin level $\leq 10 \mathrm{~g} / \mathrm{dL}$ or a $2 \mathrm{~g} / \mathrm{dL}$ decrease if abnormal at baseline; thrombocytopenia was defined as a platelet count $<$ $150,000 \mathrm{cells} / \mu \mathrm{L}$ or a $50 \%$ decrease if already low at baseline.

Estimated ranges for the primary outcome were $58 \%$ in non-obese group and $41 \%$ in obese group based on MRSA pneumonia studies by Wunderink et al. ${ }^{[20,21]}$. An estimated 2:1 ratio of non-obese to obese patients was chosen based on average prevalence of obesity in U.S. populations. Thus, a sample size of 297 patients (198 patients in non-obese group and 99 patients in obese group) was calculated with $80 \%$ power to detect a difference of $17 \%$ in each primary outcome in obese patients (BMI $\geq 30 \mathrm{~kg} / \mathrm{m}^{2}$ ) versus non-obese patients (BMI $<30 \mathrm{~kg} / \mathrm{m}^{2}$ ) with a two-tailed $\alpha$ value of 0.05 . Descriptive statistics such as mean and median were used to describe the study population. In order to detect any significant association between patient characteristics and the primary outcomes, the Pearson correlation test was used. Categorical data were evaluated using the Chi-square test or the Fisher's exact test, as appropriate. Outcomes with continuous data were evaluated with the Student's t test. All analyses were performed using Microsoft Excel 2010, SPSS ${ }^{\circledR}$ Statistical Software package version 20, and G*Power software v.3.1.7 (Franz Faul, Universität Kiel, Germany).

\section{Results}

A total of 1466 patients were identified from linezolid billing code and screened for eligibility. (Figure 1) depicts the screening and enrollment process. Due to the strict inclusion and exclusion criteria, this study did not meet its power. 1396 patients were excluded, with the two most common reasons for exclusion being lack of sufficient linezolid doses $(<6$ doses) and other MRSA infections not related to pneumonia. Of the 70 patients included, 25 patients were classified as obese and 45 patients as non-obese (approximately a 1:2 ratio). (Table 1) summarizes the baseline demographics, which were mostly comparable among the obese and non-obese groups. A total of 27 patients (19 patients in the non-obese group vs. 8 patients in the obese group) were admitted to ICU; mean SAPS II scores were 43 and 45 in the non-obese and obese groups $(\mathrm{p}=0.68)$, respectively. Only 4 patients were on mechanical ventilation in ICU; all were in the non-obese group.

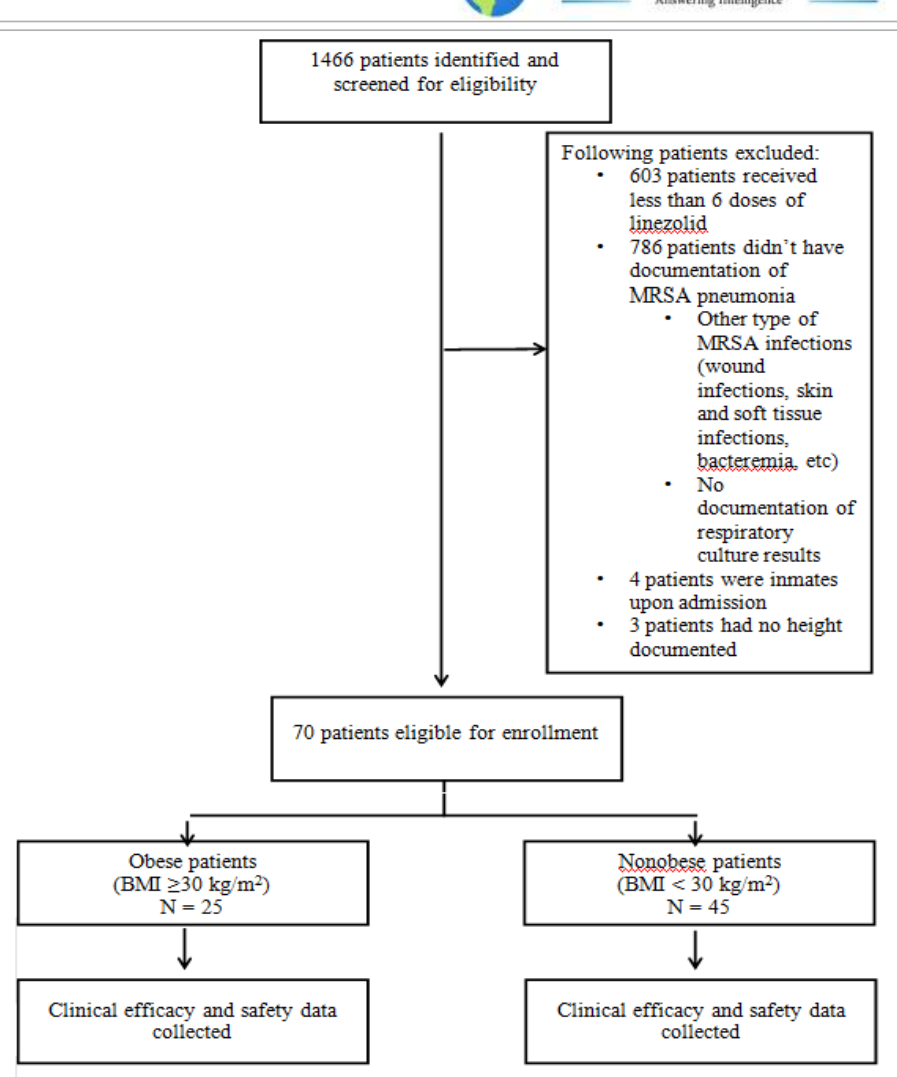

Figure 1: Patient Enrollment Process.

All patients received IV linezolid at the beginning of the course of therapy, but 10 patients in the non-obese group and 9 patients in the obese group received oral linezolid during the later course of therapy. In the obese group, 1 patient switched to a different antibiotic therapy due to thrombocytopenia and 2 patients switched to doxycycline due to co-infection (osteomyelitis). In the non-obese group, 2 patients switched to vancomycin due to co-infection (bacteremia) and 1 patient switched to doxycycline for co-infection (osteomyelitis). These patients were considered as treatment failures, since the reasons for switching therapy were due to potential toxicity or less efficacy.

Other concomitant antibiotics were evaluated. As empiric therapy, most patients received ceftriaxone and levofloxacin or azithromycin for community-acquired pneumonia (CAP). For hospital-acquired pneumonia (HAP) or healthcare-associated pneumonia (HCAP), most patients received piperacillin-tazobactam or cefepime with levofloxacin or an aminoglycoside. Use of other empiric antibiotics was not different between the obese and non-obese patients and almost all of them were discontinued after MRSA was identified from sputum culture(s) unless other co-infections were identified.

Some patients had other microorganism(s) from the respiratory culture besides MRSA. The number of mixed culture results was $11 \%$ in non-obese group vs. $20 \%$ in obese group. Concurrent antibiotics for mixed culture results were appropriate based on the culture sensitivity. One patient in non-obese group had MRSA and Pseudomonas aeruginosa co-infection which required more than 21 days of linezolid, piperacillin-tazobactam and amikacin due to multidrug resistance and was marked as therapy failure. Other mixed cultures required less duration of other antibiotic therapy than linezolid for MRSA. Therefore, clinical cure at day 21 was not affected by mixed culture results in all patients. 
Linezolid for MRSA Pneumonia: The Impact of Obesity

Table 1: Baseline Patient Demographics.

\begin{tabular}{|c|c|c|c|}
\hline Characteristic & Non-obese $(n=45)$ & Obese $(n=25)$ & p-value \\
\hline Age $($ years, mean \pm SD) & $\begin{array}{c}63.84 \pm 17 . \\
(23-88)\end{array}$ & $\begin{array}{c}58.36 \pm 10.87 \\
(41-81)\end{array}$ & 0.009 \\
\hline \multicolumn{4}{|l|}{ Gender (n,\%) } \\
\hline Female & $11(24.4 \%)$ & $15(60 \%)$ & 0.003 \\
\hline Male & $34(75.6 \%)$ & $10(40 \%)$ & \\
\hline Height $(\mathrm{cm}$, mean \pm SD) & $\begin{array}{c}174.37 \pm 11.43 \\
(137.16-195.50)\end{array}$ & $\begin{array}{c}168.66 \pm 9.50 \\
(149.86-82.88) \\
\end{array}$ & 0.0215 \\
\hline Weight $(k g$, mean \pm SD) & $\begin{array}{c}72.7 \pm 13.83 \\
(42.6-100.6) \\
\end{array}$ & $\begin{array}{l}115.9 \pm 28.15 \\
(75.6-177.2) \\
\end{array}$ & $<0.001$ \\
\hline BMI $\left(\mathrm{kg} / \mathrm{m}^{2}\right.$, mean $\left.\pm \mathrm{SD}\right)$ & $\begin{array}{l}23.79 \pm 3.75 \\
(14.7-29.3)\end{array}$ & $\begin{array}{l}43.03 \pm 11.46 \\
(30.6-67.69)\end{array}$ & $<0.001$ \\
\hline $\mathrm{SCr}(\mathrm{mg} / \mathrm{dL}$, mean $\pm \mathrm{SD})$ & $1.57 \pm 1.77$ & $2.09 \pm 2.13$ & 0.30 \\
\hline $\mathrm{CrCl}(\mathrm{ml} / \mathrm{min}$, mean $\pm \mathrm{SD})$ & $69.7 \pm 42.1$ & $182.5 \pm 74.4$ & 0.37 \\
\hline SAPSII score (mean \pm SD)* & $43.192 \pm 17.75$ & $45.105 \pm 13.33$ & 0.68 \\
\hline \multicolumn{4}{|l|}{ Type of Pneumonia (n,\%) } \\
\hline CAP & $17(37.8 \%)$ & $14(56 \%)$ & \multirow{3}{*}{0.128} \\
\hline HCAP & $13(28.9 \%)$ & $8(32 \%)$ & \\
\hline HAP & $15(33.3 \%)$ & $3(12 \%)$ & \\
\hline Mixed culture (n, \%) & $5(11.1 \%)$ & $5(20 \%)$ & 0.309 \\
\hline \multicolumn{4}{|l|}{ MIC (mcg/mL) †(n) } \\
\hline$<$ or $=1$ & 19 & 11 & \multirow{3}{*}{0.888} \\
\hline 1.5 or 2 & 24 & 13 & \\
\hline$>2$ & 2 & 1 & \\
\hline
\end{tabular}

*SAPS II score only recorded for ICU patients: nonobese group $\mathrm{n}=19$; obese group $\mathrm{n}=8$

$\dagger$ MIC for vancomycin

$\mathrm{BMI}=$ body mass index, $\mathrm{SCr}=$ serum creatinine, SAPS II Score $=$ Simplified Acute Physiology Score, $\mathrm{CAP}=$ Community-Acquired Pneumonia, $\mathrm{HCAP}=$ Healthcare-Associated Pneumonia, HAP $=$ Hospital-Acquired Pneumonia

Total duration of therapy was calculated based on the number of linezolid doses and was not different between the obese group and the non-obese group (8.64 +/- 4.18 days vs. $8.93+/-4.31$ days).

(Table 2) summarizes the results of the primary outcome and secondary outcomes. Clinical cure rate at day 21 was lower in the obese group compared to the non-obese group. A low in-hospital mortality rate $(15-20 \%)$ was observed in both groups. Incidence of anemia was higher in the non-obese group; similarly, incidence of thrombocytopenia was higher in the nonobese group.

Table 2: Primary and Secondary Outcomes.

\begin{tabular}{|c|c|c|}
\hline & Non-obese $(n=45)$ & Obese $(n=25)$ \\
\hline \multicolumn{3}{|l|}{ Primary Outcome } \\
\hline Clinical Cure Rate (n,\%) & $27(60 \%)$ & $12(48 \%)$ \\
\hline \multicolumn{3}{|l|}{ Secondary Outcomes } \\
\hline Anemia (n,\%) & $16(64 \%)$ & $14(31 \%)$ \\
\hline Thrombocytopenia (n,\%) & $10(40 \%)$ & $9(20 \%)$ \\
\hline In-hospital Mortality (n,\%) & $5(20 \%)$ & $7(15.6 \%)$ \\
\hline
\end{tabular}

\section{Discussion}

To our knowledge, this is the first study reporting clinical outcomes of linezolid therapy between obese and non-obese patients. While there is a general lack of data regarding appro- priate methods of dosing with antimicrobial agents in obesity, pharmacokinetic and pharmacodynamic (PK/PD) indices have been used to predict in vivo antimicrobial efficacy ${ }^{[10]}$. Pertinent $\mathrm{PK} / \mathrm{PD}$ indices for the oxazolidinone class of antibiotics have been reported as the time above the minimum inhibitory concentration $(\mathrm{T}>\mathrm{MIC}$ ) and the area under the concentration-time curve divided by MIC (AUC/MIC); a moderate post-antibiotic effect has also been observed ${ }^{[22]}$. Based on these indices, certain dosing strategies have been suggested for linezolid therapy in obese patients. Increasing the dosing frequency from every 12 hours to every 8 hours has been proposed for patients with isolates having an MIC of $4 \mathrm{mg} / \mathrm{L}^{[10]}$. Similarly, continuous infusions of linezolid have been shown to result in 100\% alveolar diffusion and concentrations that exceed the MIC breakpoint for Staphylococcus aureus in serum and epithelial lining fluid for $100 \%$ of the time ${ }^{[23]}$. However, these recommendations (along with previously published literature) have been mostly based on serum or plasma concentrations and associated pharmacokinetic data, with little to no focus on clinically relevant outcomes.

Previous studies regarding the impact of weight on linezolid efficacy and safety have provided conflicting data on whether serum linezolid concentrations are similar or significantly different between the obese and non-obese patient populations. Any number of factors could be responsible for this variability in data, such as patient-specific characteristics, comorbid conditions, and concurrent administration of interacting medications. With regard to pharmacokinetic parameters, 
lower linezolid concentrations in some obese patients might be explained by increased clearance, changes in absorption, or an increased volume of distribution. The post hoc analysis of phase IV clinical trial data by Puzniak et al. did not measure serum linezolid concentrations but compared clinical success rates between 224 patients with MRSA pneumonia divided into 4 weight quartiles ranging from $40 \mathrm{~kg}$ to $215 \mathrm{~kg}$, each quartile containing approximately 50 - 60 patients $^{[19]}$. Although no differences were found in clinical success rates between quartiles, this secondary analysis was primarily designed to compare vancomycin and linezolid in each weight quartile and was not powered to detect differences between quartiles in the linezolid group alone. Additionally, height was not recorded for these patients; hence, no direct comparison between obese and non-obese patients could be made. Our study was designed to make such a comparison between obese and non-obese groups regarding similar clinical outcomes.

The primary outcome in this study was chosen on the basis of its ability to reflect the clinical efficacy of antibiotics in pneumonia. Duration of MRSA pneumonia treatment is recommended for 7 - 21 days by the Infectious Diseases Society of America $^{[1]}$. Hence, clinical cure rate at day 21 was felt to be an important outcome with regard to the patient's course of treatment and overall clinical condition. In-hospital mortality as a secondary outcome was included as a measure of linezolid treatment failure, while the incidence rates of anemia and thrombocytopenia were assessed as safety outcomes due to the hematologic toxicities associated with linezolid. This study revealed $48-60 \%$ clinical cure rate and $15-20 \%$ in-hospital mortality rate with linezolid therapy. These were very similar results with the two previous linezolid studies compared tovancomycin in MRSA pneumonia by Wunderink et al. ${ }^{[20,21]}$.

The studies by Puzniak et al. and Wunderink et al. detected $22-37 \%$ and $43-55 \%$ mixed culture results ${ }^{[18-21]}$. This study revealed only $10-20 \%$ mixed culture results. This difference can be explained by the different types of pneumonia included in the studies. Puzniak et al. and Wunderink et al. only included nosocomial pneumonia. On the other hand, this study included CAP, HAP and HCAP and resulted in a higher percentage of pure MRSA cultures and correspondingly lower mixed culture results.

There were no statistically significant differences in any of the primary or secondary outcomes analyzed. This was not unexpected, as we were not able to enroll enough patients to meet prespecified power criteria. The limited sample size was most likely due to the stringent inclusion and exclusion criteria, particularly the requirement for documented MRSA-positive sputum cultures. While this was our way of ensuring that only pneumonia cases secondary to MRSA infection were included, it also severely limited patient enrollment, as many patients with pneumonia are empirically treated without obtaining a sputum sample for culture or the sputum sample obtained is of poor quality. The issue of unmet power in this study could potentially be overcome by expanding data collection to multiple institutions.

Other than the small sample size, there were two major limitations to our study. The first limitation deals with how the primary outcome was determined. Clinical cure rate at day 21 is definitely a clinically relevant outcome, as this is a composite measure of fever, respiratory rate, blood pressure, pulse, and ox- ygen saturation, thus providing a more reliable indicator of the patient's treatment progress and overall clinical condition. However, the length of time to clinical cure may prove to be a more useful outcome to assess between obese and non-obese patients. The second limitation deals with the MIC report in our institution, in regards to MRSA susceptibility. Our institution typically reports vancomycin MICs, but not linezolid MICs. Only two patients in the non-obese group and one patient in the obese group had a vancomycin MIC $>2 \mathrm{mcg} / \mathrm{mL}$. The clinical cure rate could be affected by different MIC values. A larger sample size and a quantitative MIC report would allow comparison of the clinical cure rate in different MICs as a subgroup analysis.

\section{Conclusions}

Our study results showed a $12 \%$ lower clinical cure rate of MRSA pneumonia in obese compared to non-obese patients; this should be verified in a powered study. Future studies should incorporate data from multiple institutions to facilitate a larger sample size and to ensure external validity.

Acknowledgement: The authors would like to thank David Fike, $\mathrm{Ph} . \mathrm{D}$. for his assistance with study design and statistical analysis.

\section{References}

1. Liu, C., Bayer, A., Cosgrove, S.E., et al. Clinical practice guidelines by the Infectious Diseases Society of America for the treatment of methicillin-resistant Staphylococcus aureus infections in adults and children. (2011) Clin Infect Dis 52(3): e18-55.

2. Methicillin-resistant Staphylococcus aureus (MRSA). (2016) Centers for Disease Control and Prevention.

3. Burton, D.C., Edwards, J.R., Horan, T.C., et al. Methicillin-resistant Staphylococcus aureus central line-associated bloodstream infections in US intensive care units, 1997-2007. (2009) JAMA 301(7): 727-736. 4. Kollef, M.H., Shorr, A., Tabak, Y.P., et al. Epidemiology and outcomes of health-care-associated pneumonia: results from a large US database of culture-positive pneumonia. (2005) Chest 128(6): 3854-3862. 5. National Center for Health Statistics. (2015) Centers for Disease Control and Prevention.

6. Mandell, L.A., Wunderink, R.G., Anzueto, A., et al. Infectious Diseases Society of America/American Thoracic Society consensus guidelines on the management of community-acquired pneumonia in adults. (2007) Clin Infect Dis 44Supp12: S27-S72.

7. American Thoracic Society, Infectious Diseases Society of America. Guidelines for the management of adults with hospital-acquired, ventilator-associated, and healthcare-associated pneumonia. (2005) Am J Respir Crit Care Med 171(4): 388-416.

8. Zyvox [package insert]. (2013) New York, NY: Pharmacia \& Upjohn Co.

9. Flegal, K.M., Carroll, M.D., Kit, B.K., et al. Prevalence of obesity and trends in the distribution of body mass index among US adults, 1999-2010. (2012) JAMA 307(5): 491-497.

10. Janson, B., Thursky, K. Dosing of antibiotics in obesity. Curr Opin Infect Dis. (2012) 25(6): 634-649.

11. Bhalodi, A.A., Papasavas, P.K., Tishler, D.S., et al. Pharmacokinetics of intravenous linezolid in moderately to morbidly obese adults. (2013) Antimicrob Agents Chemother 57(3): 1144-1149.

12. Hamilton, R., Thai, X.C., Ameri, D., et al. Oral bioavailability of linezolid before and after Roux-en-Y gastric bypass surgery: is dose modification necessary in obese subjects? (2013) J Antimicrob Chemother 68(3): 666-673. 
13. Stein, G.E., Schooley, S.L., Peloquin, C.A., et al. Pharmacokinetics and pharmacodynamics of linezolid in obese patients with cellulitis. (2005) Ann Pharmacother 39(3): 427-432.

14. Mihic, T., Chow, I., Mabasa, V. Linezolid Dosing in a Mordbidly Obese Patient with MRSA Pneumonia. (2016) Ann Pharmacother 50(2):154.

15. Tsuji, Y., Hiraki, Y., Matsumoto, K., et al. Evaluation of the pharmacokinetics of linezolid in an obese Japanese patient. (2012) Scand J Infect Dis 44(8): 626-629.

16. Mersfelder, T.L., Smith, C.L. Linezolid pharmacokinetics in an obese patient. (2005) Am J Health Syst Pharm 62(5): 464,467.

17. Muzevich, K.M., Lee, K.B. Subtherapeutic linezolid concentrations in a patient with morbid obesity and methicillin-resistant Staphylococcus aureus pneumonia: case report and review of the literature. (2013) Ann Pharmacother 47(6): e25.

18. Puzniak, L.A., Morrow, L.E., Huang, D.B., et al. Impact of weight on treatment efficacy and safety in complicated skin and skin structure infections and nosocomial pneumonia caused by methicillin-resistant Staphylococcus aureus. (2013) ClinTher 35(10): 1557-1570.
19. Cai, Y., Chai, D., Falagas, M.E., et al. Immediate hematological toxicity of linezolid in healthy volunteers with different body weight: a phase I clinical trial. (2012) J Antibiot (Tokyo) 65(4):175-178.

20. Wunderink, R.G., Rello, J., Cammarata, S.K., et al. Linezolid vs vancomycin: analysis of two double- blind studies of patients with methicillin-resistant Staphylococcus aureus nosocomial pneumonia. (2003) Chest 124(5):1789-1797.

21. Wunderink, R.G., Niederman, M.S., Kollef, M.H., et al. Linezolid in methicillin-resistant Staphylococcus aureus nosocomial pneumonia: a randomized, controlled study. (2012) Clin Infect Dis 54(5): 621-629. 22. Rayner, C.R., Forrest, A., Meagher, A.K., et al. Clinical pharmacodynamics of linezolid in seriously ill patients treated in a compassionate use programme. (2003) Clin Pharmacokinet 42(15): 1411-1423.

23. Boselli, E., Breilh, D., Caillault-Sergent, A., et al. Alveolar diffusion and pharmacokinetics of linezolid administered in continuous infusion to critically ill patients with ventilator-associated pneumonia. (2012) J Antimicrob Chemother 67(5): 1207-1210.
Ommega Online Publishers

Journal Title: Journal of Pharmacy \& Pharmaceutics

Journal Short Name: J Pharm Pharmaceutics
Journal ISSN: 2377-1313

E-mail: pharmacoinformatics@ommegaonline.com

Website: www.ommegaonline.org 